\title{
The eddy structure in Stokes flow in a cavity
}

\author{
By P.N. SHANKAR \\ Computational and Theoretical Fluid Dynamics Division, National Aeronautical Laboratory, \\ Bangalore 560017, India
}

(Received 22 July 1992 and in revised form 21 October 1992)

Stokes flow in a two-dimensional cavity of rectangular section, induced by the motion of one of the walls, is considered. A direct, efficient calculational procedure, based on an eigenfunction expansion, is used to study the eddy structure in the cavity. It is shown that some of the results of earlier studies are quantitatively in error. More importantly, two interesting questions, namely the extent of the symmetry of the corner eddies and their relationship to the large-eddy structure are settled. By carefully examining the rather sudden change in the main eddy structure for cavities of depth around 1.629, it is shown that the main eddies are formed by the merger of the primary corner eddies; the secondary corner eddies then become the primary corner eddies and so on. Thus, in the evolution of the large-eddy structure the corner eddies, in some sense, play the role of progenitors. This explicit prediction should be experimentally verifiable.

\section{Introduction}

We consider Stokes flow in a rectangular two-dimensional cavity in which the flow is driven by the steady motion of one of the walls (see figure 1). Our objective is to determine the motion in the cavity by an analytical or semi-analytical method. We are interested in the eddy structure in the cavity, particularly the nature of the corner eddies and their relationship to the main eddy structure.

This problem has been considered in a fine paper by Pan \& Acrivos (1967), whose primary aim, however, was an experimental investigation of the flow in the Reynolds number range 20-4000. They used a direct numerical method to determine Stokes flow in the driven cavity. Of particular interest are their determination of the remarkably symmetric corner-eddy structure for the square cavity and the dependence of the largeeddy structure on the cavity depth.

The present work was motivated by the need for an accurate solution with which the results of a Navier-Stokes code being developed could be checked. It has turned out, however, that the Stokes flow is of interest in its own right. The method used is an eigenfunction expansion procedure that is non-trivial because all the eigenvalues are complex and because no direct expansion procedure is available. First, the solution for the finite cavity is obtained. Then the solution for the infinite cavity, not treated by Pan \& Acrivos, is obtained. Surprisingly, it is found that while the solutions obtained by Pan \& Acrivos are qualitatively correct they suffer from serious quantitative errors.

The most interesting feature of this paper, however, should be the discovery of the relationship of the main eddy structure to the corner eddies. The efficient calculational procedure used here permits us to carefully investigate the changes in the eddy structure with increasing depth around the depth range where the number of large eddies increases by one. We find an aesthetically pleasing evolutionary process at work, one which leads to the formation of the large eddies from the merger of the corner 


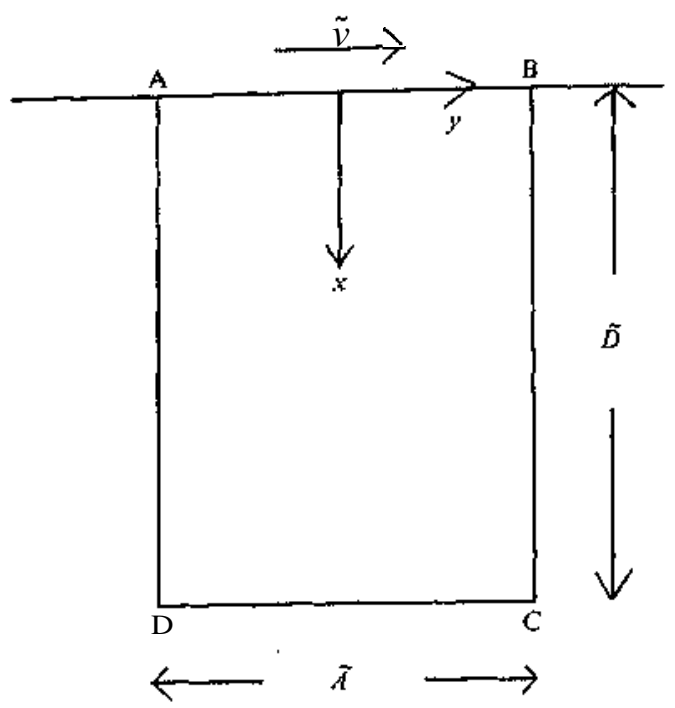

FIGURE 1. The cavity geometry.

eddies. This is the only phase during which the symmetry imposed by Moffatt's (1964) 'antisymmetric' similarity solution is broken.

For completeness were formulate the problem as follows. Let all lengths be normalized by $A$, the width of the cavity, and velocities by $V$, the speed of the driven face. Then referring to figure 1 , the Stokes flow problem in the cavity is defined by

$$
\begin{gathered}
\nabla^{\mathbf{4}} \psi=0 \text { in the cavity, } \\
\psi=0 \text { on the boundaries } \mathrm{AB}, \mathrm{BC}, \mathrm{CD} \text { and } \mathrm{DA}, \\
\psi_{x}=-^{-} \text {on } \mathrm{AB}, \psi_{x}=0 \text { on } \mathrm{DC}, \\
\psi_{y}=\wedge \text { on } \mathrm{BC} \text { and } \mathrm{AD},
\end{gathered}
$$

where $\psi$ is the dimensionless streamfunction. We shall be interested in obtaining solutions not only for finite depths $D$ of the cavity but also for infinitely deep cavities. We remark that in view of the symmetries of $(1 a)$ and the boundary conditions about $y=0$, the solution will also be symmetric about $y=0$.

\section{Eigenfunction expansion for the driven cavity}

\subsection{A cavity of finite depth}

If we assume modal solutions of the form $\psi \sim \mathrm{e}^{\lambda x} \phi(y)$, (1) implies that $\phi$ has to satisfy

$$
\begin{gathered}
\left(\mathrm{d}^{2} / \mathrm{d} y^{2}+\lambda^{2}\right)^{2} \phi=0, \\
\phi=\mathrm{d} \phi / \mathrm{d} y=0 \quad \text { at } y= \pm \frac{1}{2} .
\end{gathered}
$$

It is easy to show that the symmetric eigenfunctions satisfy the transcendental equation

and ${ }^{\top}$ are themselves given by

$$
\sin \lambda_{n}=-\lambda_{n}
$$

$$
\phi_{n}=y \sin \lambda_{n} y-\backslash \tan \left(\frac{1}{2} \lambda_{n}\right) \cos \lambda_{n} y .
$$

The roots of (3), which has been widely studied in the elasticity context (see Robbins \& Smith 1948 and Johnson \& Little 1965 for example), are all complex. The roots are 
easy to compute using Newton's method with the asymptotic forms providing starting values. It is also easy to show that if $\lambda_{n}$ is an eigenvalue so are $-\lambda_{n}$ and $\lambda_{n}$. In the following, $\lambda_{n}$ will refer to the eigenvalue in the first quadrant. Since $\psi(x, y)$ is real, an eigenfunction expansion for $\psi$ in terms of the eigenfunctions (3) must take the form

$$
\psi(x, y)=\mathrm{S}_{n=1}^{\infty}\left\{a_{n} \phi_{n} \mathrm{e}^{-\lambda_{n} x}+\bar{a}_{n} \bar{\phi}_{n} \mathrm{e}^{-\bar{\lambda}_{n} x}+b_{n} \phi_{n} \mathrm{e}^{-\lambda_{n}(D-x)}+\bar{b}_{n} \bar{\phi}_{n} \mathrm{e}^{-\bar{\lambda}_{n}(D-x)}\right\},
$$

where the $a_{n}$ and $b_{n}, n-1,2, \ldots$, have to be determined from the boundary conditions. Assuming completeness (see Gregory 1980 for some completeness results in the elasticity context), our main difficulty is that there is no known explicit procedure, based on the orthogonality or biorthogonality of the eigenfunctions, to determine the coefficients. It is true that Joseph \& Sturges (1978) have given a procedure based on a biorthogonal series expansion but the coefficients, not determined by a direct procedure, have to be solved for by truncation of the series and the solution of a linear system of equations. A number of important mathematical results on the convergence of the biorthogonal series are given in Joseph, Sturges \& Warner (1982). The results that Joseph \& Sturges present for the corner eddies for the $D=5.0$ case are very inaccurate because of the use of too small a number of eigenfunctions. The difficulties are directly overcome in this study by recourse to a direct, least-total-error-squared procedure. It will be shown in what follows that very accurate solutions can, indeed, be obtained.

Let us truncate expression (5) to the first $N$ terms. Choose $m$ equidistant points on $0 \leqslant y<0.5$ and determine the error made in the satisfaction of the boundary conditions at $x=0$ and $x=D$, At $x-0$, if $e_{1}(y)$ and $e_{2}(y)$ are the errors made at $y$ in satisfying $(1 b)$ and $(1 \mathrm{c})$ respectively and if $E_{1}^{2}$ and $E_{2}^{2}$ are the totals over the $m$ points of the squares of the errors, we have

$$
\begin{aligned}
& e_{1}(y)=\sum_{n=1}^{N} \phi_{n}(y)\left\{a_{n}+b_{n} \mathrm{e}^{-D \lambda_{n}}\right\}+\bar{\phi}_{n}(y)\left\{\bar{a}_{n}+\bar{b}_{n} \mathrm{e}^{-D \bar{\lambda}_{n}}\right\}, \\
& e_{2}(y)=-1+{\underset{n}{n=1}}_{\mathrm{S}}^{N}\left[\lambda_{n} \phi_{n}(y)\left\{a_{n}-b_{n} \mathrm{e}^{-D \lambda_{n}}\right\}+\bar{\lambda}_{n} \bar{\phi}_{n}(y)\left\{\bar{a}_{n^{-}} \bar{b}_{n} \mathrm{e}^{-D \bar{\lambda}_{n}}\right\}\right], \\
& E_{1}^{2}={\underset{j=1}{m}}_{j=1}^{m} e_{1}^{2}\left(y_{j}\right), \quad E l={\underset{j=1}{m}}_{j=1}^{m} e_{2}^{2}\left(y_{j}\right) .
\end{aligned}
$$

Similarly at $x=D$ we compute the errors to be

$$
\begin{gathered}
e_{3}(y)=\sum_{n=1}^{N}\left[\phi_{n}(y)\left\{a_{n} \mathrm{e}^{-\lambda_{n} D}+b_{n}\right\}+\bar{\phi}_{n}(y)\left\{\bar{a}_{n} \mathrm{e}^{-\bar{\lambda}_{n} D}+\bar{b}_{n}\right\}\right] \\
e_{4}(y)=\sum_{n=1}^{N}\left[\lambda_{n} \phi_{n}(y)\left\{a_{n} \mathrm{e}^{-\lambda_{n} D}-b_{n}\right\}+\bar{\lambda}_{n} \bar{\phi}_{n}(y)\left\{\bar{a}_{n} \mathrm{e}^{-\bar{\lambda}_{n} D}-\bar{b}_{n}\right\}\right], \\
E_{0}=\sum_{j=1}^{m} e_{3}^{2}\left(y_{j}\right), \quad E l=\int_{j=1}^{m} e_{4}^{2}\left(y_{j}\right) .
\end{gathered}
$$

The aim now is to minimize the total of the sums of the squares of the errors, $E_{\mathrm{T}}^{2}\left(E_{\mathrm{T}}^{2}=E_{1}^{22}+E_{2}^{2}+E_{3}^{2}+E_{4}^{2}\right)$, and to this end we set

$$
\frac{\partial E_{T}^{2}}{\partial a_{n \mathrm{r}}}=\frac{\partial E_{T}^{2}}{\partial a_{n \mathrm{i}}}=\frac{\partial E_{T}^{2}}{\partial b_{n \mathrm{r}}}=\frac{\partial E_{T}^{\rho}}{\partial b_{n \mathrm{i}}}=0 \text { for } n=1,2, \ldots, N,
$$




\begin{tabular}{|c|c|c|c|}
\hline & $D=0.25$ & $D=1.0$ & $D=5.0$ \\
\hline $\begin{array}{l}a_{\mathrm{R}} \\
b_{\mathrm{I}}\end{array}$ & $\begin{array}{c}1.22-4.58 \times 10^{-1} \mathrm{i} \\
-7.81 \times 10^{-1}+4.43 \times 10^{-1} \mathrm{i}\end{array}$ & $\begin{array}{r}5.12 \times 10^{-1}-9.73 \times 10^{-2} \mathrm{i} \\
-2.68 \times 10^{-2}+9.73 \times 10^{-3} \mathrm{i}\end{array}$ & $\begin{array}{l}5.11 \times 10^{-1}-9.68 \times 10^{-2} \mathrm{i} \\
1.41 \times 10^{-9}-5.64 \times 10^{-10} \mathrm{i}\end{array}$ \\
\hline$a_{2}$ & $\begin{array}{r}-1.49 \times 10^{-1}-9.26 \times 10^{-3} \mathrm{i} \\
4.55 \times 10^{-2}+1.00 \times 10^{-2} \mathrm{i}\end{array}$ & $\begin{array}{l}-1.47 \times 10^{-1}+1.28 \times 10^{-2} \mathrm{i} \\
-2.64 \times 10^{-9}+1.64 \times 10^{-3} \mathrm{i}\end{array}$ & $\begin{aligned}- & 1.48 \times 10^{-1}+1.29 \times 10^{-2} \mathrm{i} \\
& 1.07 \times 10^{-10}-7.71 \times 10^{-11} \mathrm{j}\end{aligned}$ \\
\hline $\begin{array}{l}a_{3} \\
b_{3}\end{array}$ & $\begin{array}{l}6.97 \times 10^{-2}+3.35 \times 10^{-3} \mathrm{i} \\
-2.1 \times 10^{-3}-7.73 \times 10^{-3} \mathrm{i}\end{array}$ & $\begin{array}{l}7.63 \times 10^{-2}-1.31 \times 10^{-3} \mathbf{i} \\
3.94 \times 10^{-4}+5.32 \times 10^{-5} \mathbf{i}\end{array}$ & $\begin{aligned} & 7.63 \times 10^{-2}-1.31 \times 10^{-3} \mathrm{i} \\
- & 1.93 \times 10^{-11}-7.40 \times 10^{-14} \mathrm{i}\end{aligned}$ \\
\hline $\begin{array}{l}a_{4} \\
b_{4}\end{array}$ & $\begin{array}{l}-4.56 \times 10^{-2}-1.80 \times 10^{-3} i \\
-2.77 \times 10^{-3}+3.09 \times 10^{-3} \mathrm{i}\end{array}$ & $\begin{array}{l}-4.83 \times 10^{-2}-1.24 \times 10^{-3} \mathrm{i} \\
-7.23 \times 10^{-5}-5.37 \times 10^{-5} \mathrm{i}\end{array}$ & $\begin{array}{r}-4.83 \times 10^{-2}-1.25 \times 10^{-3} \mathrm{i} \\
4.16 \times 10^{-12}+1.99 \times 10^{-12} \mathrm{i}\end{array}$ \\
\hline $\begin{array}{l}a_{5} \\
b_{5}\end{array}$ & $\begin{array}{l}3.29 \times 10^{-2}+1.76 \times 10^{-4} \mathrm{i} \\
2.14 \times 10^{-3}-8.86 \times 10^{-4} \mathrm{i}\end{array}$ & $\begin{array}{l}3.40 \times 10^{-2}+1.85 \times 10^{-3} \mathrm{i} \\
1.40 \times 10^{-5}+2.57 \times 10^{-5} \mathbf{i}\end{array}$ & $\begin{array}{r}3.40 \times 10^{-2}+1.86 \times 10^{-3} \mathrm{i} \\
-1.03 \times 10^{-12}-1.10 \times 10^{-12} \mathrm{i}\end{array}$ \\
\hline $\begin{array}{l}a_{51} \\
b_{51}\end{array}$ & $\begin{array}{r}4.26 \times 10^{-4}+4.23 \times 10^{-4} \mathrm{i} \\
-1.53 \times 10^{-i}+1.35 \times 10^{-8} \mathrm{i}\end{array}$ & $\begin{array}{r}4.26 \times 10^{-4}+4.23 \times 10^{-4} \mathrm{i} \\
-1.88 \times 10^{-9}-1.78 \times 10^{-9} \mathrm{i}\end{array}$ & $\begin{array}{l}4.26 \times 10^{-4}+4.23 \times 10^{-4} \mathrm{i} \\
1.08 \times 10^{-16}+5.76 \times 10^{-17} \mathrm{i}\end{array}$ \\
\hline $\begin{array}{l}a_{52} \\
b_{52}\end{array}$ & $\begin{array}{r}-3.87 \times 10^{-4}-4.10 \times 10^{-4} \mathrm{i} \\
1.46 \times 10^{-7}-9.35 \times 10^{-9} \mathrm{i}\end{array}$ & $\begin{array}{r}-3.87 \times 10^{-4}-4.10 \times 10^{-4} \mathrm{i} \\
1.77 \times 10^{-9}+1.71 \times 10^{-9} \mathrm{i}\end{array}$ & $\begin{array}{l}-3.87 \times 10^{-4}-4.10 \times 10^{-4} \mathrm{i} \\
-1.03 \times 10^{-16}-5.46 \times 10^{-17} \mathrm{i}\end{array}$ \\
\hline $\begin{array}{l}a_{\mathrm{s3}} \\
b_{53}\end{array}$ & $\begin{array}{r}3.50 \times 10^{-4}+3.97 \times 10^{-4} \mathrm{i} \\
-1.39 \times 10^{-7}+6.18 \times 10^{-8} \mathrm{i}\end{array}$ & $\begin{array}{r}3.50 \times 10^{-4}+3.97 \times 10^{-4} \mathrm{i} \\
-1.66 \times 10^{-9}-1.64 \times 10^{-9} \mathrm{i}\end{array}$ & $\begin{array}{l}3.50 \times 10^{-4}+3.97 \times 10^{-4} \mathrm{i} \\
9.73 \times 10^{-17}+5.12 \times 10^{-17} \mathrm{i}\end{array}$ \\
\hline $\begin{array}{l}a_{54} \\
b_{54}\end{array}$ & $\begin{array}{r}-3.16 \times 10^{-4}-3.83 \times 10^{-4} \mathrm{i} \\
1.34 \times 10^{-7}-3.53 \times 10^{-9} \mathrm{i}\end{array}$ & $\begin{array}{r}-3.16 \times 10^{-4}-3.83 \times 10^{-4} \mathrm{i} \\
1.57 \times 10^{-9}+1.58 \times 10^{-9} \mathbf{i}\end{array}$ & $\begin{array}{l}-3.16 \times 10^{-4}-3.83 \times 10^{-4} \mathrm{i} \\
-9.43 \times 10^{-17}-4.81 \times 10^{-17} i\end{array}$ \\
\hline $\begin{array}{l}a_{55} \\
b_{55}\end{array}$ & $\begin{array}{r}2.83 \times 10^{-4}+3.69 \times 10^{-4} \mathrm{i} \\
-1.29 \times 10^{-7}+5.01 \times 10^{-10} \mathbf{i}\end{array}$ & $\begin{array}{r}2.83 \times 10^{-4}+3.69 \times 10^{-4} \mathrm{i} \\
-1.49 \times 10^{-9}-1.54 \times 10^{-9} \mathrm{i}\end{array}$ & $\begin{array}{l}2.83 \times 10^{-4}+3.69 \times 10^{-4} \mathrm{i} \\
9.15 \times 10^{-17}+4.69 \times 10^{-17} \mathrm{i}\end{array}$ \\
\hline
\end{tabular}

where $a_{n \mathrm{r}}$ and $a_{n \mathrm{i}}$ are the real and imaginary parts of $a_{n}$, etc. This leads to a system of $4 N$ linear algebraic equations for the $4 N$ real coefficients $a_{n \mathrm{r}}, a_{n \mathrm{n}}, b_{n \mathrm{r}}$ and $b_{n \mathrm{i}}$. The solution of this linear system is routine.

It should be pointed out that the $a_{n}$ and $b_{n}$ should be left free to be determined by the above procedure: any attempt to arbitrarily eliminate the $b_{n}$ in terms of $a_{n}$ by forcing $\psi=0$ or $\partial \psi / \partial x=0$ termwise at $x=0$ or $D$ will lead to failure.

All computations were done using complex double-precision arithmetic. For some of the cavity depths considered, i.e. for $\mathrm{D}=1.0$ and 5.0, the coefficients were computed with $N=50,75,100,125$ and 150 (and $m=N$ ); it was found that the coefficients were very rapidly convergent in $N$. For all the tables presented here the data pertain to the $n=100$ case. The 10 pairs of coefficients $\mathrm{fl}_{1}-\mathrm{a}_{\mathrm{B}}, \mathrm{a}_{61}-\mathrm{a}_{\mathrm{B}} 5$ and $b_{1}-b_{5}, b_{51}-b_{55}$ are tabulated in table 1 for $D=0.25,1.0$ and 5.0 respectively. For clarity only three significant figures are displayed even though they have been computed to more than twice that accuracy. A crucial issue is that of how well the boundary conditions at $x=0$ and $x=\mathrm{D}$ are satisfied. An idea of the nature and magnitude of the errors made in the satisfaction of the boundary data can be gained from figure 1 . Only the range $0.4 \leqslant v<0.5$ is shown since the errors are so much smaller, sometimes orders of magnitude smaller, for the range $0 \leqslant y<0.4$. Note that the ordinate has been magnified in the figures to the left, i.e. for $0.4 \leqslant y \leqslant 0.45$, since the errors decay very rapidly away from the singularity at $y=0.5$. As expected the errors are greatest near this point but these are greatly reduced by increasing the number of eigenfunctions from 50 to 100. Generally, it is found that the errors are larger near the upper wall $(x=0)$ and that the errors on the lower wall decay rapidly with depth. It is to be expected that the discontinuity in $-\psi_{x}$ at $y- \pm 0.5$ will impede the convergence of the solution. Note that this singularity will affect any solution procedure including a direct 


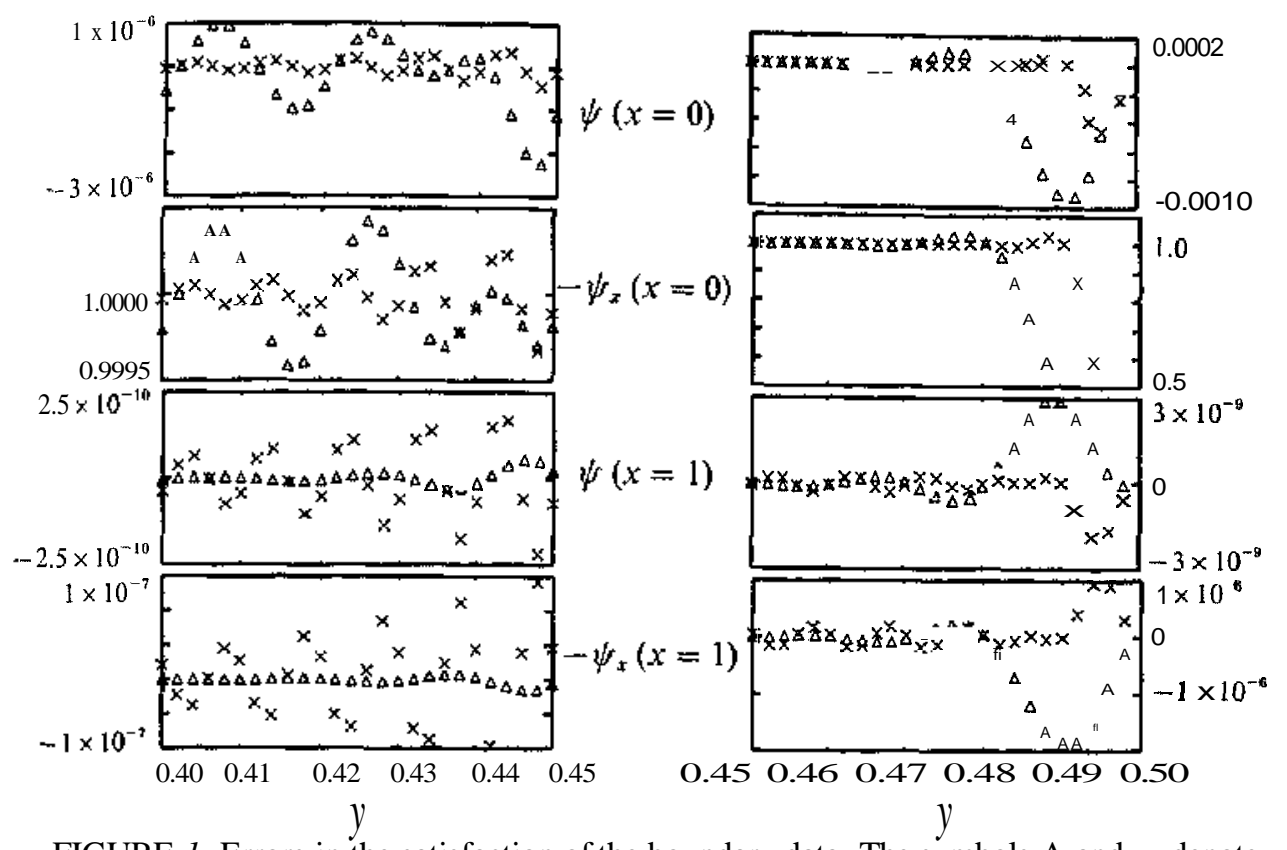

FIGURE 1. Errors in the satisfaction of the boundary data. The symbols A and $\mathrm{x}$ denote computations with 50 and 100 eigenfunctions respectively.

finite difference procedure. Detailed studies were carried out to determine the effects of increasing $N$ on the satisfaction of the boundary conditions. It was found that away from the corner the boundary conditions were accurately represented fairly soon. In the neighbourhood of the corner, however, a larger and larger number of eigenfunctions are required to better represent the boundary conditions as the corner is approached. Note that the eigenfunction expansion necessarily forces $v=-\psi_{x}$ to vanish at $y=0.5$, no matter how large $N$ is. We emphasize once again that the discontinuity in the boundary condition at $y= \pm 0.5$ will cause difficulties no matter what calculational procedure is used.

Finally, it should be emphasized that although comparatively large numbers of eigenfunctions are needed for accuracy in the neighbourhood of the upper corners of the cavity, the solution converges very rapidly everywhere else. Thus if only 50 eigenfunctions are used the results would be indistinguishable from all the results using 100 eigenfunctions. This is an important practical consideration.

\subsection{The infinitely deep cavity}

For the infinitely deep cavity, $D \rightarrow \infty$, it is natural to omit the terms corresponding to $\exp \left(\lambda_{n} x\right)$ in (5) on grounds of boundedness, since $\operatorname{Re}\left(\lambda_{n}\right)>0$ for all $n$. We thus seek an eigenfunction expansion of the form

$$
\psi(x, y)={\underset{n=1}{\infty}}_{n=1}^{\infty}\left\{a_{n} \phi_{n}(y) \mathrm{e}^{-\lambda_{n} x}+\bar{a}_{n} \bar{\phi}_{n}(y) \mathrm{e}^{-\bar{\lambda}_{n} x}\right\},
$$

where only the coefficients $a_{n}$ have to be determined from the conditions $\psi=0$ and $v=1$ at $\mathrm{A}-=0$. The least total-error-squared procedure of $\$ 2.1$ can again be applied but now only $E_{1}^{2}$ and $E_{2}^{2}$ need be considered. The computations are simpler since only $2 N$ equations have to be solved for the real and imaginary parts of the $N$ coefficients $a_{n}$.

The ten coefficients $a_{1}-a_{5}, a_{51}-a_{55}$ are shown to three significant figures in table 2 . 


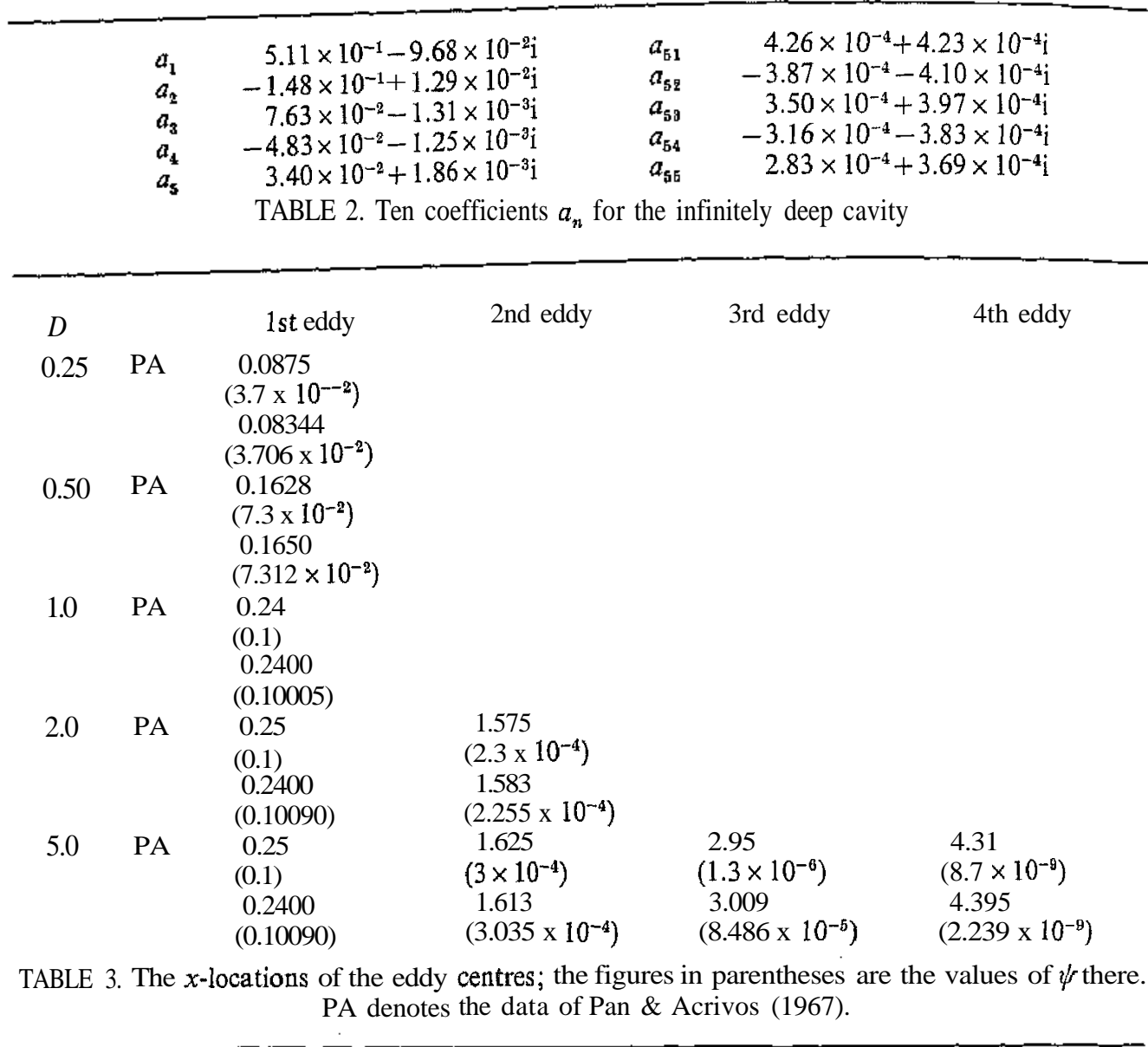

\section{The overall flow field}

\subsection{The large-eddy structure}

A qualitative picture of the eddy structure as a function of cavity depth has already been well brought out by Pan \& Acrivos (1967, to be referred to as PA henceforth), who made computations for $D=0.25,1,2$ and 5 . In general it was known that the number of large eddies in the cavity increased with depth $D$, e.g. for $D=0.25,0.5$ and 1 there was one eddy, two when the depth was 2 and four when the depth was 5 . We can now more precisely state that the ranges $D \leqslant 1.6,1.7 \leqslant D \leqslant 3.0,3.1 \leqslant D \leqslant 4.4$, $4.5 \leqslant \mathrm{D} \leqslant 5.8$ and $5.9 \leqslant \mathrm{D} \leqslant 7.2$ correspond to flow fields with $1,2,3,4$ and 5 main eddies respectively. Note that each eddy settles to a depth dimension of about 1.3. Table 3 lists the locations of the centres of the large eddies as given by the present computations for the values of D considered by PA; also given are the magnitudes of $\psi$ at these locations. Comparing with PA, we see that the eddy centre locations compare well for moderate $D$ but discrepancies appear for small and large $D$ especially for the third and fourth eddies. Similar trends appear as regards the magnitudes of $\psi$ at the eddy centres, except that the discrepancies are more serious, especially for the lower eddies.

In order to account for these discrepancies let us first recall the methods of computation used. PA used a direct numerical, relaxation procedure with the finest 
(a)

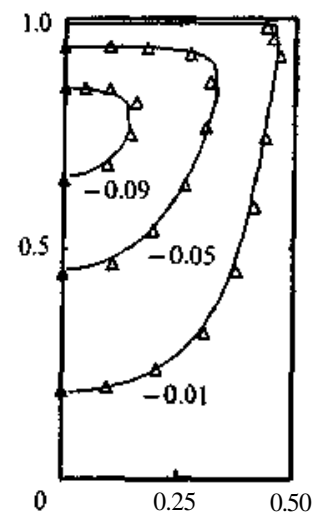

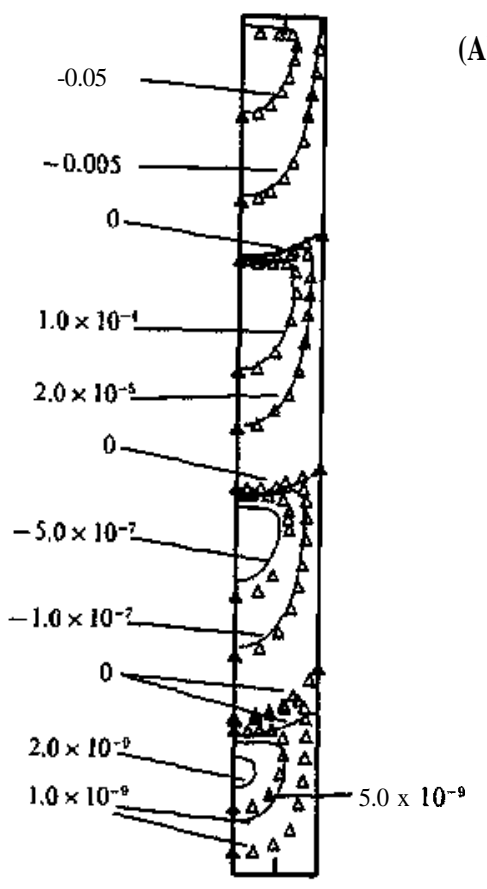

(A)

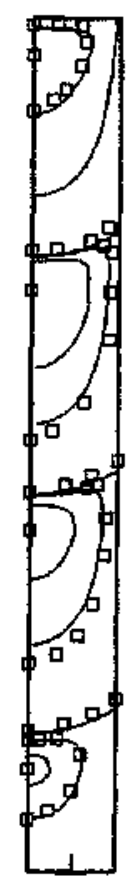

FIGURE 3. The main or large-eddy streamline patterns for $(a) D=1.0$ and $(b)$ 5.0. The numbers refer to the streamfunctionvalues. A, Data of Pan \& Acrivos (1967); $\square$ data of Joseph \& Sturges (1978); —, present data.

mesh size being 0.0125 for $D=0.25$ and $0.5,0.01$ for $D=1.0$, and 0.025 for $D=2.0$ and 5.0. The corners were treated separately, but we shall come to that later. In the present eigenfunction expansion procedure the field equation $\left(\nabla^{4} \psi=0\right)$ is satisfied exactly, everywhere in the field, the boundary conditions at $y=+0.5$ are satisfied exactly; only the boundary conditions at $x=0$ and $D$ are satisfied approximately, but increasingly accurately as $N$ increases (as shown in §2.1). Because of obvious storage limitations the finite difference calculation utilizes a coarser grid precisely when the grid needs to be finer, i.e. for increasing $D$. It therefore seems most probable that the results in PA for the lower large eddies, the difficult ones to resolve, are inaccurate because of an inadequately fine grid. Round-off errors are also likely to more seriously affect the finite difference calculation than the eigenfunction expansion. Note, for example, that PA had to solve a system of almost 10000 linear equations for $D-1.0$, while the present procedure requires the solution of only 200 and 400 linear equations for $N=50$ and 100 respectively. The finite differencecalculation will also, of course, suffer from truncation errors. On the other hand, we have shown by systematically increasing $N$ from 50 to 150 that there is hardly any change in the eddy structure. We therefore conclude that the discrepancies are due to errors in the finite difference computation. One might guess that the situation will be even worse for the more delicate corner eddies and this is indeed so, as will be shown.

The streamlines for the square cavity and the cavity of depth 5 are shown in figure 3. Note the large errors in the streamfunction values for the lower eddies in the data of PA. It is surprising that the results of Joseph \& Sturges (1978) with 20 eigenfunctions for $D=5.0$ are as good as they are. They are seriously in error only near the eddy boundaries. Joseph \& Sturges give no results for any other depths, but the present calculations suggest that their errors would increase considerably for smaller depths. 

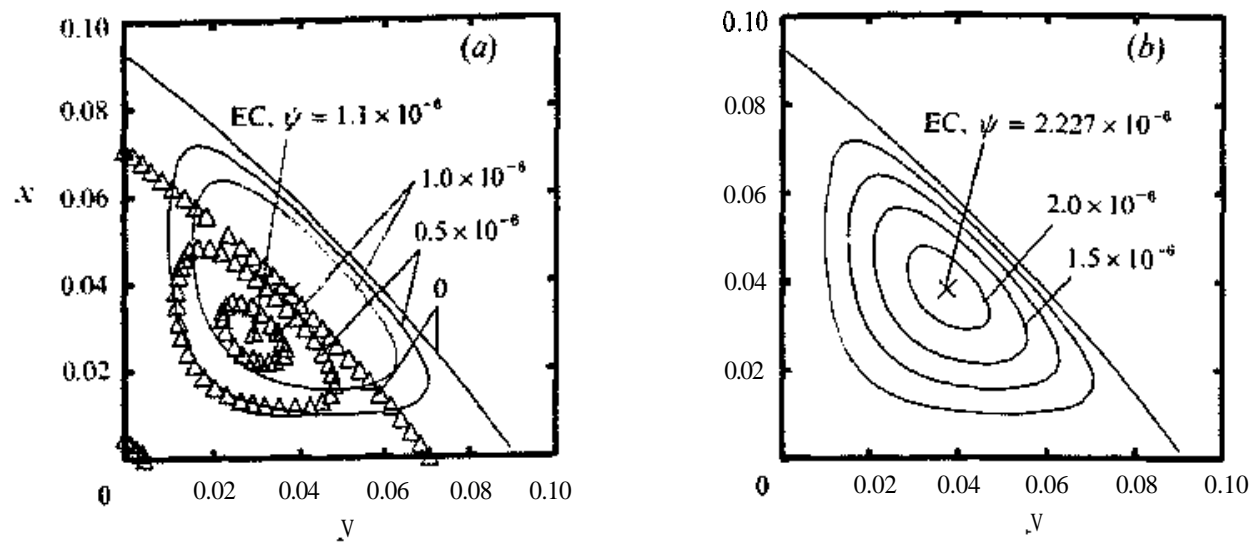

FIGURE 4. The primary corner eddy for $D=1.0$. The data from Pan \& Acrivos are shown by the symbol $\mathrm{A}$ in $(a)$. EC denotes eddy centre.

Twenty eigenfunctions are really insufficient to adequately represent the boundary data. This can be confirmed by examining their table 1 ; for example the error at $. \mathrm{x}=0$ on $v$ is greater than $10 \%$ at $\mathrm{v}=0.35$, while with the present computation it is of the order of $0.0002 \%$.

\subsection{The corner eddies}

The most interesting feature of driven cavity flows is the complex corner-eddy structure at the lower corners of the cavity. Employing a general similarity analysis, Moffatt (1964) had shown that the flow near the corner must consist of a sequence of eddies of decreasing size and decreasing intensity. For the square cavity PA confirmed this prediction by computing the detailed structure of the first three corner eddies. A particularly striking feature of these solutions was the remarkable symmetry of the flow fields about the bisector of the corner; in fact, the eddy centres lie almost exactly on the bisector. An obvious question that arises is: how accurate is this symmetry?

Figure 4 shows the details of the corner-eddy structure for $D=1$ according to the present computations. Figure $3(a)$ shows, for the primary corner eddy, comparisons with the results of PA. Although qualitatively similar, there are large discrepancies with regard to both the size and intensity of the eddy: by the present calculations the eddy's linear dimension is approximately 9.2/7.2 as large and twice as intense as that predicted by PA. These large discrepancies are surprising since with a mesh size of 0.01 about 40 mesh points should lie within the eddy; perhaps this number is insufficient. Another possible source of error is the method used by PA to resolve the eddy structure: keeping the solution exterior to the eddy fixed at the 'converged' state the eddy region was subdivided into finer and finer meshes and the eddy solution iterated on these. This procedure, although possibly sufficient for a qualitative picture of the eddy structure, is not sufficient for accurate quantitative predictions. In any case I believe the results of the present computations to be far more accurate and substantially correct. Some more support for the claim is provided by the fact that to at least three significant figures the eddy centre location and strength are unchanged by increasing $N$ from 50 to 150 . PA also display results for the secondary and tertiary corner eddies for $D=1$. But we believe that these computations are conceptually flawed; it is not possible to reliably resolve structures finer than the mesh size used for the main flow field simply by freezing it and recomputing the fine structure with a fine grid. In essence what PA have done is to solve Moffatt's problem numerically. Resolution is a fundamental difficulty that affects the present calculations, too, as these 

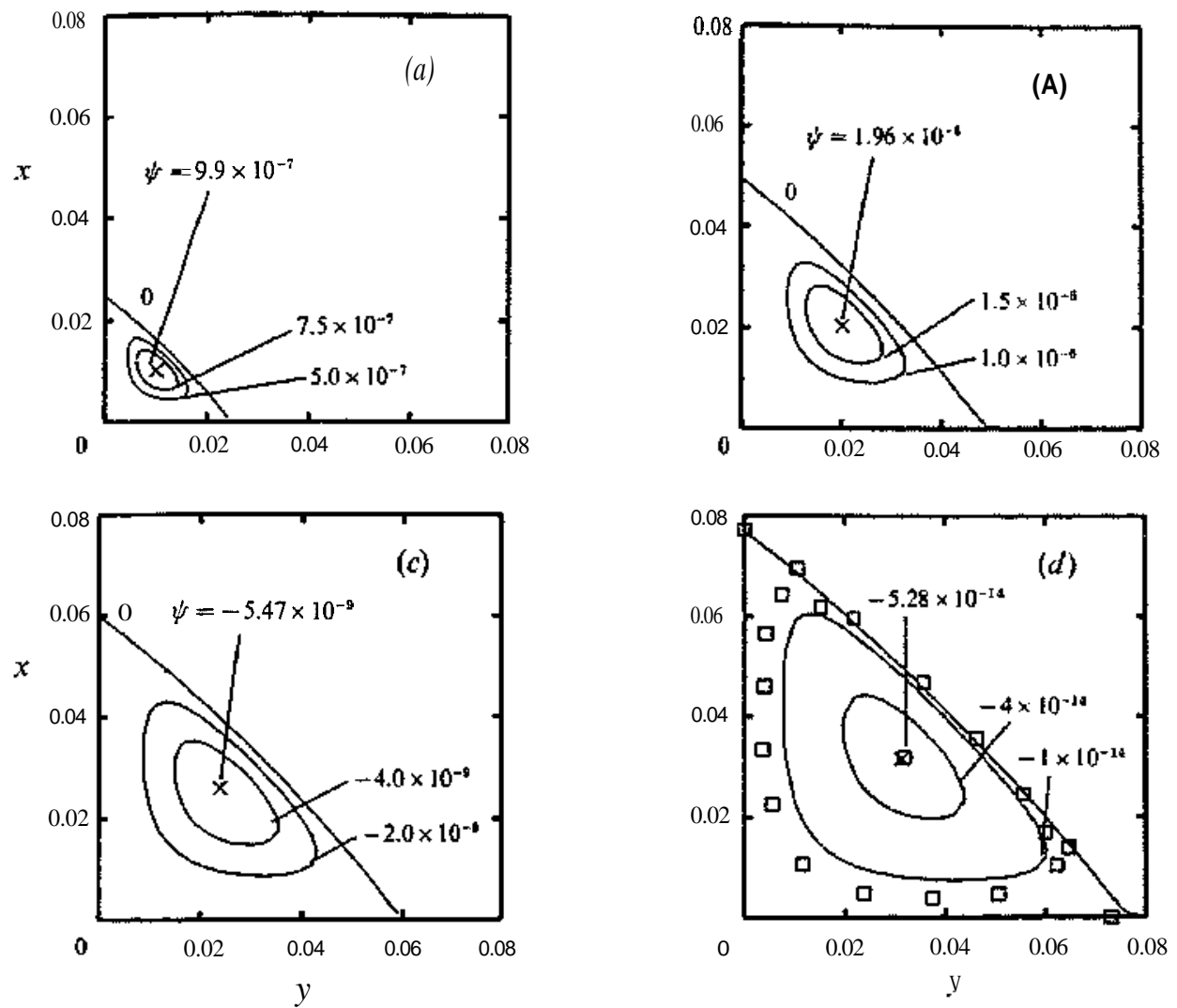

FIGURE 5. Primary corner eddies for various depths $D$. (a) $D-0.25,(b) 0.5$, (c) $2.0,(d)=5.0$. Data of Joseph \& Sturges are shown by the symbol $\square$ •

also suffer errors at the boundaries. While the primary corner eddies can be resolved accurately, the secondary ones cannot be resolved reliably unless the primary corner eddy becomes large and strong.

As indicated earlier Joseph \& Sturges (1978) present results for the $D=5.0$ case using just 20 eigenfunctions. The corner eddies that they find, while qualitatively satisfactory, are quantitatively very inaccurate. For example, the peak value of ty appears to be in error by at least a factor of 2. The reason for this is that 20 eigenfunctions are really quite inadequate for capturing such details of the flow field. This is not surprising when one examines the large errors that they report on the satisfaction of the boundary data. $\dagger$

Figure 5 shows the corner eddies for $D=0.25,0.5,2.0$ and 5.0. Note that while the size of the eddy changes, the remarkable approximate symmetry is maintained. Thus the Moffatt similarity solution dominates the corner flow field.

t At the suggestion of a referee, computations for $D=5.0$ using only 20 eigenfunctions were carried out in order to compare them with the results of Joseph \& Sturges (1978) as given in their table 1. With the present method the maximum errors (tested at 100 points in $0 \leqslant y \leqslant 0.5)$ in $|\psi(0, y)|$, $|v(0, y)|$ and $|\psi(5, y)|$ were $2.262 \times 10^{-3}, 1.647 \times 10^{-13}$ and $5.512 \times 10^{-15}$ which compare with Joseph \& Sturges' $2.08 \times 10^{-4}, 9.046 \times 10^{-9}$ and $3.083 \times 10^{-16}$ (tested at 20 points). However, for the crucial $v(0, y)$ the maximum overshoot is 0.03 while Joseph \& Sturges quote 0.264 . Equally important, the oscillations decay very rapidly towards the centre of the cavity in these calculations while with the Joseph \& Sturges computations comparatively large oscillations persist to the centre. So the present method seems to be at least as accurate as that of Joseph \& Sturges for this set of data. 


\begin{tabular}{ccccc}
\hline 1st eddy & 2nd eddy & 3rd eddy & 4 th eddy & 5th eddy \\
0.2400 & 1.6127 & 3.0088 & 4.4050 & 5,8002 \\
$(0.10090)$ & $\left(3.0354 \times 10^{-4}\right)$ & $\left(8.486 \times 10^{-7}\right)$ & $\left(2.372 \times 10^{-9}\right)$ & $\left(6,632 \times 10^{-12}\right)$ \\
6 th eddy & 7 th eddy & 8 th eddy & 9 th eddy & 10 th eddy \\
7.1960 & 8.5925 & 9.9876 & 11.383 & 12.780 \\
$\left(1.854 \times 10^{-14}\right)$ & $\left(5.183 \times 10^{-17}\right)$ & $\left(1.449 \times 10^{-19}\right)$ & $\left(4.051 \times 10^{-22}\right)$ & $\left(1.132 \times 10^{-24}\right)$ \\
& TABLE 4. Eddy centre locations and $\psi_{\max }$ values for $D=\infty$ &
\end{tabular}

\subsection{The infinite cavity}

We observe that because of storage limitations a finite difference computation cannot handle very deep cavities, let alone cavities of infinite depth. The present procedure leads to a rapid solution for the latter case. Since qualitatively the flow field is little different from the case of deep cavities, we only present the data on the first 10 eddies in table 4 . Comparing with table 3 we note that as $D$ increases, the eddy parameters rapidly converge to their asymptotic values at $D=\infty$.

\section{Evolution of the eddy structure with cavity depth}

With the normalization chosen the flow field under study depends on, apart from the Reynold's number, a single parameter alone, the depth $D$ of the cavity. Thus the flow field should change, if at all, only with the depth. Figures 3 and 5 and other data presented so far show how the main and corner eddy structures change with the depth. While it is clear that in general the number of large eddies increases with increasing depth, the origin of this structural change and its relationship to the corner-eddy structure is still unclear. A related issue is the question of the remarkable symmetry of the corner-eddy structure; all the data presented so far indicate that this approximate symmetry persists no matter how unsymmetric the main flow field is about the bisector of the corner angle. But surely this cannot always hold. We will now investigate these two issues; it is apparent that the best range of $D$ to study these issues in is one where the number of primary eddies increases by one. We shall therefore narrow our scrutiny to the neighbourhood of $D=1.6$.

Figure 6 shows how the corner-eddy pattern changes when $D$ is increased systematically from 1 to 1.6. The corner-eddy size and strength increase in this range, with the increase particularly rapid towards the end of the range. Thus both the linear dimension and the strength change by a factor of almost 5 in response to a depth change from 1.0 to 1.6 . Note, however, that the increase in $\psi_{\max }$ is not monotonic; between depths of 1 and $1.3 \psi_{\max }$ actually decreases. We observe that though the departure from symmetry is clearly indicated by the visible differences between the $x$ and $>$ ' lateral dimensions (figure $6 d$ ), the centre of the eddy still continues to lie very close to the bisector of the corner.

Around a depth of 1.6 it now becomes obvious that the growing corner eddies will have to touch if the growth continues. Indeed, while at $D=1.6295$ the two primary corner eddies are still distinct, at a depth of 1.62975 they have already touched and begun to merge. Figure $7(a, b)$ shows the initiation of the merger process; note that only half the cavity is shown as the field is symmetric about the midplane. The departures from symmetry are now clearly evident and thus, finally, the hold of the similarity solution on the corner-eddy structure is broken. (A referee has pointed out 

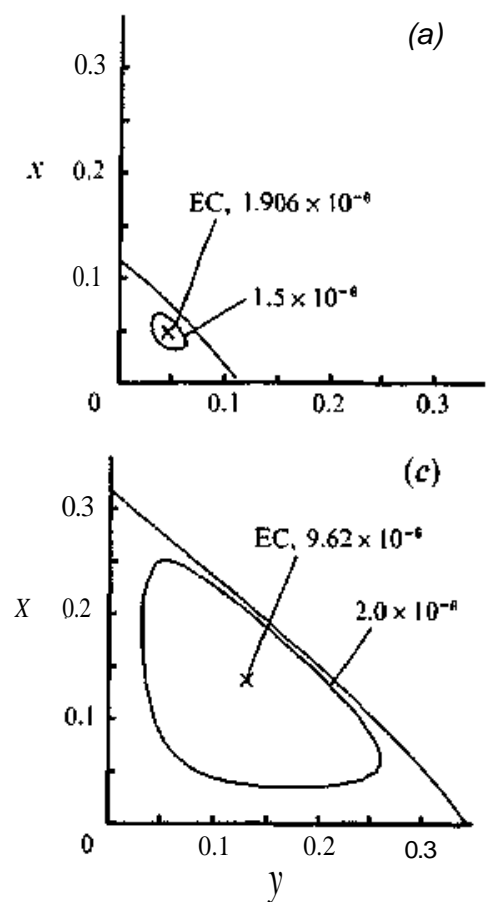
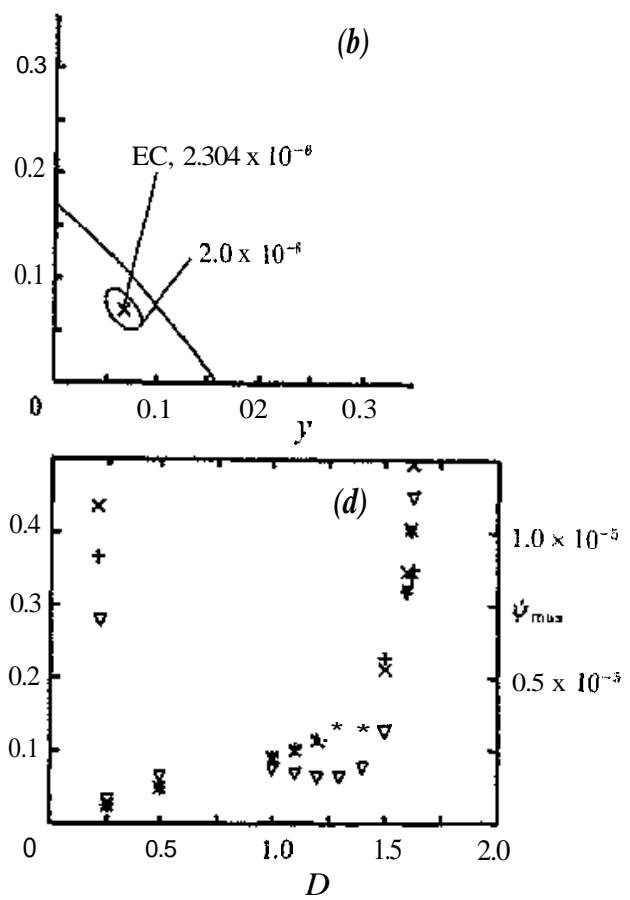

FIGURE 6. The growth of the primary corner eddy with increasing depth beyond $D=1.0$ : $(a)$ $D=1.2,(b) 1.4,(c)$ 1.6. (d) The variation of $\psi_{\max }(\mathrm{V})$, the magnitude of the streamfunction at the eddy centre, and $l_{x}(+)$ and $l_{y}(\times)$, the linear dimensions of the corner eddy, with $D$.

that this may be viewed as being due to a larger contribution to the structure from the other Moffatt solution, his 'symmetric' one.) When $D=1.64$ (figure $7 b$ ) the process of the merger of the two primary corner eddies is clear. With the appearance of another stagnation point at the saddle-type stationary point for $\psi$, the picture is now clear: the two corner eddies merge to produce the new secondary main or large eddy. The new stagnation point located on the cavity centreline is the centre of the new large eddy. Note that the newly formed secondary main eddy is weaker than the two corner eddies by almost two orders of magnitude. We also note that the original secondary corner eddies can now be easily resolved because their strengths have increased with the increasing strength of the primary corner eddy.

In the middle stages of the evolutionary process, figure $7(c, d)$, the centre of the secondary main eddy lifts off and increases in strength while the centre of the former primary corner eddy moves towards the centre and, relatively, loses strength. It is now clear that the former primary corner eddy has become a 'free' eddy with closed streamlines forming a cat's-eye pattern. We note that this is an example (of finite extent) of what Jeffrey \& Sherwood (1980) call a region of 'blocked' flow, where the flow turns back on itself in order to move back to the other half of the cavity, enclosing in the process a region of closed streamlines. Note that as predicted by Jeffrey \& Sherwood there is no stagnation line, at least as far as we can tell. Meanwhile, the former secondary corner eddies, now the primaries of the current flow field, continue to grow.

The final stages of the evolution of the secondary main eddy are shown in figure $7(e, f)$.When $D=1.795$ the strengths of the free eddy and the secondary main eddy are almost equal and the centres are very close together; the region of closed streamlines in 

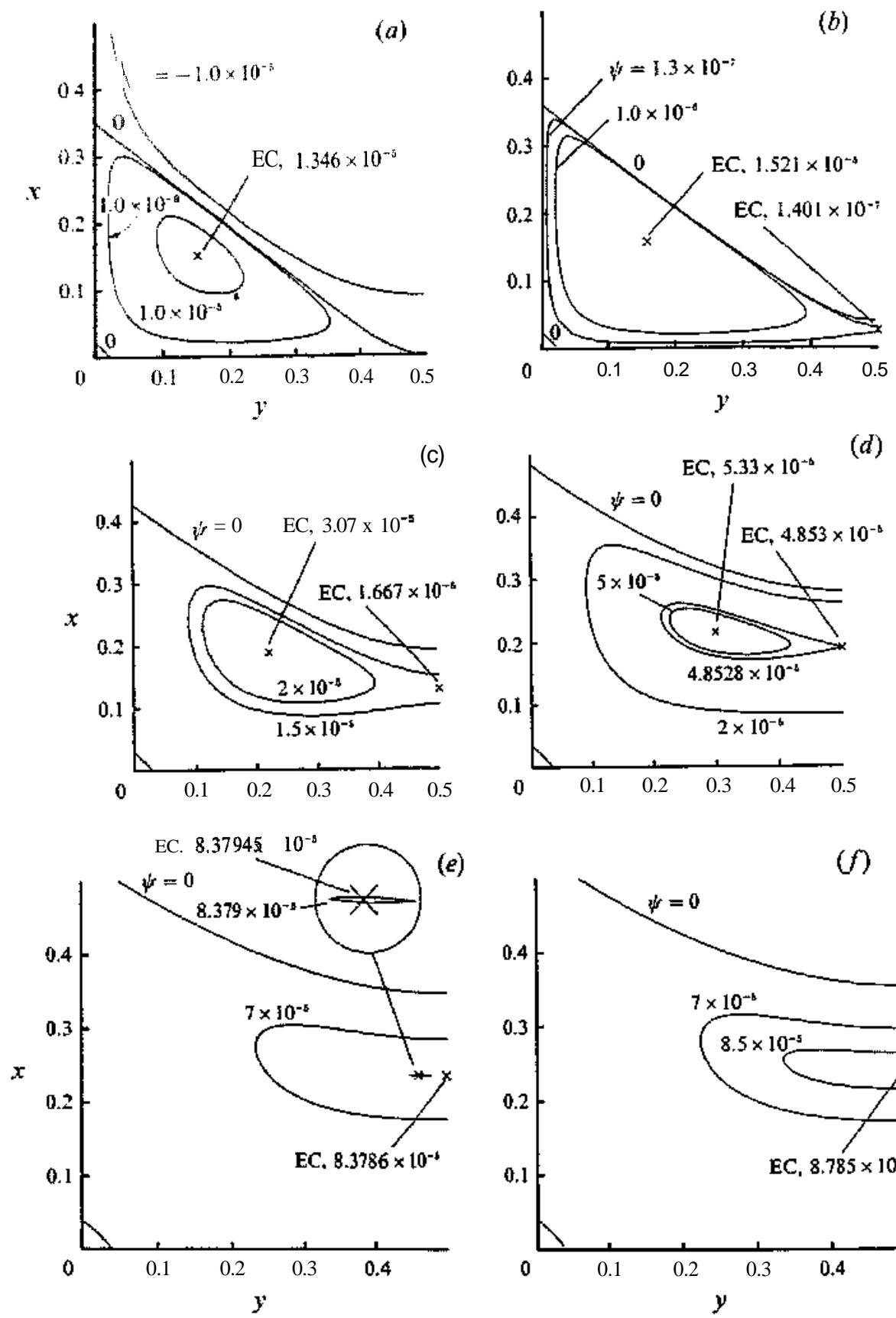

(e)

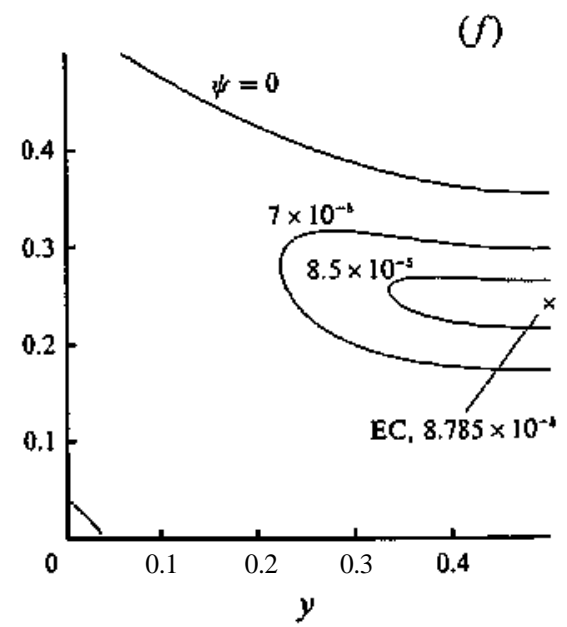

FIGURE 7. The evolution of the secondary main eddy from the primary corner eddy with increasing $D$. $E C$ denotes the eddy centres. (a) $D=1.6295$, (b) 1.64 : note the corner eddies at the lower left corners of each figure, (e) $\mathrm{D}=1.70,(d) 1.75$ : note the free eddy in the cat's eye pattern. $(e) D=1.795$, (f) 1.80: the final stages of the evolution. 
the free eddy is now very small. For $D=1.8$, the free eddy has disappeared or merged with the secondary main eddy whose strength has risen to $8.785 \times 10^{-5}$. With increasing depth, now, the qualitative picture will appear to be unchanging. This completes the evolutionary scenario that we sought.

\section{Conclusion}

In conclusion, we have shown how the secondary main eddy evolves from the merger of the two primary corner eddies. The evolution is through the formation of two free or blocked eddies which ultimately merge with the secondary main eddy. In the meanwhile the former secondary corner eddies grow and take on the roles of the new primaries. This picture has been shown in detail for the formation of the secondary main eddy; but it requires little imagination to see that all the subsequent main eddies are formed by the same type of process. Thus we might reasonably say that the corner eddies are in a sense the source of all the main eddies other than the primary. We conclude by pointing out that we could not have obtained this detailed picture of the evolution of the eddy structure if we did not have at hand an efficient computational tool such as the one developed here.

I would like to thank a referee for pointing out a number of errors in the first version of this paper. I gratefully acknowledge financial support from the Aeronautical Research \& Development Board for NAL project CF-1-120, under which this work was completed.

\section{REFERENCES}

GREGORY, R. D. 1980 The semi-infinite strip $x \geqslant 0,-1 \leqslant y \leqslant 1$; completeness of the Papkovich-Fadle eigenfunctions when $\phi_{x x}(0, y), \phi_{y y}(0, v)$ are prescribed. J. Elast. 10, 57.

JEFFREY, D. J. \& SHERWOOD, J. D. 1980 Streamline patterns and eddies in low-Reynolds-number flow. J. Fluid Mech. 96, 315.

JOHNSON, M. W. \& LITTLE, R. W. 1965 The semi-infinite elastic strip. Q. Appl. Maths 22, 335.

JOSEPH, D. D. \& STURGES, L. 1978 The convergence of biorthogonal series for biharmonic and Stokes flow edge problems: Part II. SIAMJ. Appl. Maths 34, 7.

JOSEPH, D. D., STURGes, L. D. \& WARNER, W. H. 1982 Convergence of biorthogonal series of biharmonic eigenfunctions by the method of Titchmarsh. Arch. Rat. Mech. Anal. 78, 223.

MOFFATT, H. K. 1964 Viscous and resistive eddies near a sharp corner. J. Fluid Mech. 18, 1.

PAN, F. \& ACRIVOS, A. 1967 Steady flows in rectangular cavities. /. Fluid Mech. 28, 643 (referred to herein as PA).

ROBBINS, C. I. \& SMITH, R. C. T. 1948 A table of roots of $\sin z=-$ s. Phil. Mag 39, 1004. 